\title{
PROBLEMAS ACTUALES DE LA INCAPACIDAD PERMANENTE
}

\author{
Paz Hidalgo Bermejo \\ Letrado de la Administración de la Seguridad Social
}

En julio de 2004 la Seguridad Social abonó un total de 7.880.620 pensiones contributivas en todo el territorio nacional, de los que fueron por Incapacidad Permanente 815.936, un 2,2\% más que en el ejercicio 2003, incremento muy superior al del resto de las prestaciones (por ejemplo el incremento en Jubilación fue $0,3 \%$ o de un $1,4 \%$ en Viudedad).

Para el pago de las mismas destinó la Seguridad Social del total, 4.547,89 millones de euros, 543,32 millones a la Incapacidad Permanente, frente a los 3.000,53 millones dedicados a la nómina mensual de jubilación o a los 924,36 millones de euros destinados a las pensiones de viudedad.

Estos datos ponen de manifiesto una primera desigualdad, hay más perceptores de prestación de incapacidad y se paga menos.

Junto a esta desigualdad en la cuantía y número de personas respecto de las restantes prestaciones, también existe una clara desigualdad en el importe de las pensiones de I. Permanente por Comunidades Autónomas.

Las pensiones medias de Incapacidad Permanente alcanzan en el País Vasco su importe máximo 867,78 euros mensuales, 843,78 euros en Asturias, 535,38 en Extremadura y, el importe más bajo el de 541,32 euros en Murcia.

Además se trata, la incapacidad, de la prestación contributiva que presenta mayores índices de litigiosidad. Un $27 \%$ de los expedientes tramitados acaba siendo revisado en el Orden jurisdiccional social. Esta litigiosidad, dada la actual configuración del modelo judicial, provoca una creciente y peligrosa disparidad de criterios judiciales en la interpretación y aplicación de la regulación de esta prestación. Disparidad que no es corregida mediante el mecanismo creado para tal efecto, pues 
de todos es sabido el alarmante criterio restrictivo fijado por el Tribunal Supremo en el llamado juicio de igualdad que como requisito ha fijado el legislador en la regulación del Recurso de Casación para la Unificación de Doctrina (artículo 217 de la Ley de Procedimiento Laboral) y que en la práctica ha mantenido a la Incapacidad Permanente al margen de la doctrina unificada, aun cuando no está excluida en la ley rituaria. No existe un cierre absoluto y apriorístico al recurso de casación para la Unificación de doctrina, por razón de la materia, pero son escasísimos los supuestos en los que ha prosperado el instrumento impugnatorio de referencia.

No se han producido en estos últimos años cambios profundos en el régimen jurídico de la Incapacidad Permanente, a diferencia de lo que, respecto de todo el sistema, ha ocurrido con otras prestaciones como las de jubilación y muerte y supervivencia.

Es la I. Permanente, la prestación del sistema de Seguridad Social que menos modificaciones legislativas ha experimentado en su regulación sustancial. Se suprimió la llamada Invalidez Provisional (Ley 42/94), se reguló el procedimiento de calificación y revisión (RD 1.300/95 y OM de 18-1-96), incluso se reguló la duración del procedimiento a efectos del silencio negativo y se residenciaron en el Equipo de Valoración de Incapacidades las competencias de las Unidades de Valoración Médica de Incapacidades (antes dependientes de los servicios de salud, hoy dependientes de las Comunidades Autónomas).

Los cambios sí que han afectado a la regulación de esta prestación en los regímenes especiales de autónomos y de los trabajadores por cuenta propia de otros regímenes. Efectivamente, con las últimas reformas se ha producido una extensión de la acción protectora por Incapacidad Temporal en estos regímenes, no sólo derivada de la construcción del riesgo profesional en el Régimen Especial de Trabajadores Autónomos, sino de la equiparación de su protección con la del régimen general, así como de la extensión de algunos grados de la I.P. de la que estaban excluidos.

Por otro lado, la nueva regulación derivada de las reformas introducidas en otras prestaciones relacionadas con la I.P. han supuesto novedades en su régimen jurídico. Por ejemplo, los diversos tipos de jubilación (flexible, parcial, prolongación de la jornada más allá de los 65 años) han llevado a regular las consecuencias prestacionales del trabajo en tales supuestos y sus efectos sobre la I.P.

Existe pues como segunda conclusión una regulación histórica que apenas ha sido modificada.

A pesar de su denominación (I. Permanente) la protección que genera es variable al quedar muchas veces expectante de una ulterior revisión 
por agravación o mejoría en función de la evolución y, con una repercusión diversa sobre la relación laboral, ya sea la hasta ahora excepcional de suspensión con reserva de puesto de trabajo (artículo 48-2 del E.T.), ya sea la general de extinción del contrato (artículo 49-1 del E.T.).

El Pacto de Toledo que da lugar al Acuerdo sobre Consolidación del Sistema de Seguridad Social aprobado por el pleno del Congreso de los Diputados en su sesión de 6-4-95 respecto de la I. Permanente establecía que «se mantiene como hasta ahora» $\mathrm{y}$, que el reconocimiento de la pensión en sus diferentes grados se efectuará en función de una lista en la que se fije la graduación correspondiente que será aprobada por vía reglamentaria previo informe del INSS. Esta medida, se dice, tiene como finalidad introducir la necesaria seguridad jurídica.

Tras los acuerdos de 1996 se intenta un gran cambio en la regulación de la I.P. nada menos que referida al concepto de la Incapacidad Permanente (artículo 136 de la LGSS) y, a la forma de determinación de sus grados (artículo 137 de la LGSS), reforma que queda vacía de contenido ante la ausencia de desarrollo reglamentario al que quedó condicionada su entrada en vigor, según estableció la Disposición Transitoria quinta Bis de la LGSS y, que pese a la ampliación del plazo, acordado por la Disposición Adicional 39 de la Ley 50/98, de 30 de diciembre, al día de hoy no se ha llevado a cabo.

La lista de enfermedades cuya inclusión reglamentaria dispuso la Ley 24/97, de 15 de julio, de Consolidación y Racionalización del Sistema de Seguridad Social trataba de articular un sistema de subsunción directa y automática, similar al que se contenía en el Reglamento de Accidentes de Trabajo de 22 de junio de 1956 o parecido al que se dice que se aplica actualmente en Alemania.

A pesar de que constantemente se habla del carácter profesional de la Incapacidad Permanente lo cierto es que las exigencias laborales de la actividad laboral a considerar, las características de la «profesión habitual», se dan por supuestas, máxime dado el amplio criterio interpretativo que sobre la profesión habitual han venido realizando los Tribunales superando la concreción a un determinado puesto de trabajo con la de grupo o categoría profesional del trabajador.

Predominan los criterios médicos sobre los profesionales y buena muestra de lo dicho viene dado por la tendencia a la objetivación de la valoración y así ya estamos acostumbrados a que parecidas «dolencias» obtienen igual valoración con independencia de las profesiones que desempeñan los beneficiarios. Ejemplo son la valoración del cuadro de artrosis lumbares (según se padezca afectación neurológica y disminución de la movilidad será IPT o no); la artrosis en caderas (según haya precisado implantación de una o dos prótesis totales de caderas se cali- 
ficará IPT o IPA); pérdida de visión (perdida la visión de un ojo, según sea la del otro mayor o menor al 50\% se reconocerá IPT o IPA); enfermedades cardiológicas, en las que según sea la fracción eyección menor o no al $40 \%$ se reconocerá IPA o IPT; dolencias como epilepsia que sólo cuando se califica como gran mal amparan el reconocimiento de la IPA, dolencias psíquicas para las que sólo el diagnóstico de depresión mayor o padecer síntomas sicóticos cronificado, con dos años de antigüedad y tratamiento en su diagnóstico, justifican el reconocimiento de una IPA. Los supuestos a mencionar no tienen fin y son claros ejemplos de la existencia de pautas interpretativas que siguen los Juzgados obviando la profesión habitual.

Por último, los continuos problemas interpretativos que surgen en la regulación de la prestación de Incapacidad Permanente son resueltos primero por la Entidad Gestora de la prestación y, posteriormente por la Jurisdicción social completando una regulación insuficiente que, además se reforma continuamente para asumir y, también corregir, las doctrinas jurisprudenciales.

Ejemplo de lo dicho vienen constituidos por la llamada teoría del paréntesis a efectos de acreditar el período de carencia y de determinación de la base reguladora, la revisión de la incapacidad, plazo y fijación de la entidad responsable en el pago cuando se suceden las aseguradoras, cálculo de la base reguladora, etc., y que después analizaremos de forma detallada.

Cuando la Incapacidad Permanente deriva de una previa situación de prórroga de Incapacidad temporal (sin cotización) o de paro involuntario sin percibir prestaciones, la tramitación del procedimiento de I.P. puede incidir en el cumplimiento de los requisitos de carencia.

Para atenuar perjuicios a los beneficiarios, primero la Seguridad Social (en su resolución de fecha 10-2-90 en relación con la Invalidez Provisional), y después la Jurisprudencia construyó la llamada teoría del paréntesis que aplicado al período de carencia determinó que su cómputo se retrotrajera al momento en el que se agota la obligación de cotizar (TS sentencias de 12-11-92, 9-10-95, 26-1-98...) y, finalmente se ha recogido en la Ley 52/03, de medidas específicas de Seguridad Social, cuyo artículo 13 modifica, añadiendo dos párrafos, el artículo 138 de la Ley General de la Seguridad Social.

En cuanto a la aplicación de la doctrina del paréntesis para la determinación de la base la jurisprudencia ha venido ignorando el mecanismo de integración de bases mínimas (previsto en el artículo 140 de la LGSS), acudiendo también para el cálculo de la base reguladora, a la retroacción del hecho causante al momento en que se produce el evento, a la fecha del accidente o, al momento de la determinación de la enferme- 
dad. Son exponentes de esta línea jurisprudencial las sentencias del Tribunal Supremo de 7-2-00 y las posteriores de 16 y 25 de mayo de 2000, 14 y 27 de junio de 2000, 17 y 18 de julio de 2000, 27 de noviembre de 2000, 4, 5, 7 y 10 de diciembre de 2000, 5 y 14 de febrero de 2001, 13 de marzo, 16 de mayo, 14 de junio, 18 de septiembre, 5 de noviembre y 14 de diciembre de 2001 .

Justificó el Tribunal Supremo su razonamiento en que este criterio es «más beneficioso en el cálculo de la base reguladora», afirmación que no siendo ajustada a la práctica cotidiana hizo que el propio Tribunal Supremo «rectificara» su criterio, y así en la posterior sentencia de 1 de octubre de 2002 estableció que «la solución del paréntesis no es siempre la más beneficiosa». Es a partir de este momento cuando se pone límite a la extensión de la doctrina del paréntesis a cualquier situación de falta de cotización (para obviarla en el período de cálculo de la base reguladora) y queda limitada a la situación de prórroga de Incapacidad Temporal (la regulada en el artículo 131 de la LGSS).

La Ley 52/03 altera este estado de cosas al remitir expresamente en estos casos al artículo 140 de la Ley General de la Seguridad Social, es decir, a la aplicación en el cálculo de la base reguladora de la integración con bases mínimas en los períodos correspondientes a situaciones asimiladas al alta sin obligación de cotizar. Se pone así fin a una interpretación alejada de la literalidad de la norma como se pronunció el Informe del CES de 26-3-03 al anteproyecto de ley de Disposiciones Específicas en Materia de Seguridad Social.

También y en cuanto al cálculo de la base reguladora de la Incapacidad permanente en relación con los trabajadores a tiempo parcial y fijos discontinuos, los períodos en los que no existían cotizaciones se tomaban en el importe de la base mínima pero a tiempo parcial. Los términos de la regulación existente eran claros y concretos (RDL 15/98, RD 144/99 y art. 7 del RD 1.131/02). Pese a la claridad de la norma, ésta fue reiteradamente ignorada por los Tribunales acudiendo a una interpretación «lógica» decían que «se llegaba al absurdo por que resultaba de peor condición quien tenía cotizaciones reales aun parciales que quien tenía laguna total» ${ }^{1}$.

Este criterio jurisprudencial se había ya incorporado añadiendo un párrafo al artículo 140.4 de la LGSS por el artículo 14 de la Ley 52/03, de 10 de diciembre.

1 Esta doctrina se «matiza» por el Tribunal Supremo que en doctrina unificada adopta otra solución. Pese a que reconoce que del tenor literal de las normas se deriva que sólo puedan integrarse las lagunas de cotización cuando la no obligación de cotizar abarque meses completos «de su contexto, espíritu y finalidad». 
En cuanto a la revisión de las Incapacidades, la redacción del artículo 143 de la LGSS obligaba a la fijación de la fecha de revisión pero sólo se regulaba «cuando en la resolución se reconociera pensión o revisión», por ello los Tribunales han venido impidiendo la fijación de plazo de revisión cuando la resolución administrativa fuera denegatoria de pensión, posibilitando así la continúa reproducción de expedientes de I. Permanente. Es exponente de esta línea la sentencia del TS de 30-6-00 (AS 6281).

Corrige esta interpretación el legislador mediante el artículo 15 de la Ley 52/03 que modifica el artículo 143 de la LGSS para permitir que las resoluciones del INSS en las que no se reconozca o modifique grado puedan igualmente fijar la fecha de revisión.

Tendremos que esperar a que, según esta costumbre de «corregir» las interpretaciones jurisprudenciales contrarias a la regulación y práctica administrativa, el legislador nos sorprenda con la superación de las doctrinas jurisprudenciales actuales contrarias a la interpretación y práctica administrativa, y de las que vamos a limitarnos a citar las más recientes:

La revisión de una Incapacidad Permanente Total derivada de accidente de trabajo y que por agravación, por concurrencia de una patología común, pasa a ser calificada como Incapacidad Permanente Absoluta. El Tribunal Supremo aun cuando confirma la exigencia administrativa referente al cumplimiento del requisito de carencia establecida, para la prestación de incapacidad por contingencia común, sin embargo, mantiene como base reguladora la correspondiente a la incapacidad de etiología profesional. En concreto la sentencia de 18 de febrero de 2002 (recurso 2.424/01) así lo establece basándose en un principio de interpretación a favor del beneficiario, estableciendo como causa que: «porque la base reguladora de la nueva prestación de acuerdo con las normas que rigen la Incapacidad Permanente derivada de contingencia común sería excesivamente perjudicial». Es decir sólo por un principio pro beneficiario.

Sobre la revisión por agravación de invalidez permanente de accidente de trabajo cuando concurren patología de origen común declarándose nuevo grado y el reparto de responsabilidades entre aseguradoras. Se dedica al análisis de este tema la sentencia del Tribunal Supremo, de fecha 1 de diciembre de 2003, dictada en el recurso de casación 8/4268/02. La misma parte de la ausencia de previsión legal para resolver la imputación de responsabilidades entre la aseguradora del primer accidente y la de la Entidad que asume la contingencia co- 
mún. Ni el artículo 143 de la LGSS , ni el RD 1.300/95, de 21 de julio, ni los artículos 17, 18 y 19 de la Orden Ministerial de 18 de enero de 1996 regulan esta materia. Frente al criterio de la Seguridad Social por el que se distribuye la responsabilidad entre las Mutuas o, entre el INSS y la Mutua aseguradora del primer accidente en función del importe de la base reguladora, acude el Tribunal Supremo, para crear su solución, no a la valoración conjunta de las patologías, de las derivadas de AT y de las derivadas de contingencia común, sino a la ajeneidad de unas patologías y otras y porque «en el sistema español de Seguridad Social la protección por accidentes se establece con una técnica próxima a la del aseguramiento privado».

Esta doctrina es la que también aplica el Tribunal Supremo, porque existe laguna normativa, al supuesto de agravación de una incapacidad permanente derivada de accidente tras sufrir un segundo accidente que determina el reconocimiento de una incapacidad permanente en superior grado y, existiendo cambio de aseguradoras. En este caso también se mantiene la responsabilidad, por lo antes dicho, de la Mutua aseguradora del segundo accidente que genera la nueva incapacidad (sentencia del TS, de fecha 26-5-03, dictada en el recurso 8/1846/02).

Sobre el cálculo de la base reguladora de la incapacidad Permanente Parcial. La reciente sentencia del Tribunal Supremo de fecha 29 de abril de 2004 (RJ 4362) modifica la interpretación que, del artículo 7 y 13 del Decreto 1.646/72, de 21 de junio, viene realizando el INSS.

El artículo 9 del Decreto 1.646/72, fija el contenido de la prestación en: «una cantidad a tanto alzado equivalente a veinticuatro mensualidades de la base reguladora que haya servido para determinar la prestación económica por incapacidad laboral transitoria (hoy incapacidad temporal) de la que deriva la invalidez». De otro lado el artículo 13 de la misma norma, al regular la cuantía del subsidio de Incapacidad Temporal lo fija en «el resultado de dividir el importe de la base de cotización del trabajador del mes anterior... por el número de días a que dicha cotización se refiera» (si el salario es diario) porque para las retribuciones de carácter mensual el número 2 introduce como excepción la división por treinta.

Conocido el importe de la base diaria según la forma de cálculo que con claridad establece el artículo 13 citado, la labor interpretativa se ha de realizar respecto de lo que no está regulado y no es otra cosa que la forma de calcular la mensualidad a que se refiere el artículo 7, que multiplicado por 24 da lugar al importe de la I.P. Parcial. Acudiendo al principio de proporcionalidad entre cotización y prestación, el INSS realiza este cálculo multiplicando la base diaria por el número de días 
de un año (365) y dividiendo por el número de meses del año (12). Sin embargo y basándose en ese mismo principio de proporcionalidad, el Tribunal Supremo realiza una diferenciación en función del beneficio que pueda obtener el trabajador y así para el caso de salarios mensuales multiplica la base de cotización del mes anterior a la incapacidad temporal, multiplicándola por 12 . Se aleja así del tenor de la norma pero se dice con la finalidad de hacerla coincidir con el salario realmente percibido que se dice es la intención del legislador.

Sobre la situación de alta o asimilada que como condición para causar derecho a las prestaciones, exige el artículo 124.1 de la LGSS, los tribunales han venido realizando una interpretación flexibilizadora. Sin embargo la situación actual supone la inaplicación del artículo 124 cuando de trabajadores enfermos se trata, es decir, presumiblemente de todos aquellos que instan la prestación de incapacidad permanente.

La sentencia del Tribunal Supremo de fecha 10 de noviembre de 2003 dictada en el recurso de casación para la Unificación de doctrina 8/1308/02, llega al extremo de considerar la inscripción como demandante de empleo como un «mero formalismo enervante, carente de finalidad en cuanto a la obtención de un posible empleo» y, en consecuencia, para quien está enfermo concurre la situación de asimilada al alta porque carece de finalidad la inscripción como demandante de empleo.

\section{Sobre cuál ha de entenderse como profesión habitual a efectos de la incapacidad permanente}

El artículo 137.2 de la Ley General de la Seguridad Social en su redacción anterior a la Ley 24/97, de acuerdo con lo dispuesto en la Disposición Transitoria quinta bis de la citada Ley General de la Seguridad Social, establece que debe entenderse como profesión habitual aquella a la que el trabajador dedicaba su actividad fundamental durante los doce meses anteriores a la fecha en la que se hubiera iniciado la incapacidad temporal de la que se deriva la Invalidez.

Sin embargo y pese al tenor de la norma el Tribunal Supremo en su sentencia de fecha 9-12-02 fija la profesión habitual a efectos de valoración como aquella «ejercida prolongadamente y no la residual a cuyo ejercicio ha podido haber conducido la situación invalidante».

La situación a análisis es importante dada la posibilidad de que los servicios médicos de empresa declaren no apto al trabajador para el ejercicio de su puesto de trabajo y, se acuerde, en consecuencia, el acoplamiento a otro puesto compatible con su estado clínico (en los términos del artículo 25 de la Ley de Prevención de Riesgos Laborales). Con esta situación es del todo punto relevante conocer cuál es la profesión a 
valorar a efectos de incapacidad, puesto que la actuación de la empresa no impide que el trabajador, si concurren las circunstancias de hecho y de derecho que lo permita, pueda solicitar la prestación de incapacidad que corresponda, al ser éste un derecho y no una obligación del trabajador. Asimismo y como posibles alternativas al acoplamiento, también podrá ver suspendido el contrato por encontrarse en situación de Incapacidad Temporal o, porque la empresa haga uso de lo dispuesto en el artículo 52 del Estatuto de los Trabajadores.

Volviendo al concepto de profesión habitual, es en casos como el descrito en el que, pese a tenor del artículo 137 de la LGSS, se considera como profesión habitual no la profesión que desempeña por el acoplamiento, sino la desempeñada en el resto de su vida laboral. Esta doctrina que sería coherente con el desempeño de esta última profesión por tiempo inferior a los 12 meses que fija la LGSS puede extenderse por períodos muy superiores.

El uso abusivo de tal maniobra con el amparo de la doctrina unificada ha llevado a que los TSJ hayan «cercado» la profesión conectando la norma con la doctrina unificada. No aplican los 12 meses que dice el artículo 137 pero sí exigen que el desempeño del nuevo puesto deba ser temporal. Se acude así al módulo de la duración de la Incapacidad Temporal, incluida la prórroga hasta 30 meses, como «paréntesis» en ese cómputo de los doce meses que fija la Ley General (sentencia del TSJ de Cantabria de 1-10-04).

Esta conclusión aunque pueda resulta beneficiosa para quienes no aptos para un trabajo han sido «recolocados» en otro, que ha de suponerse más liviano, sin embargo resulta claramente contraria con la propia doctrina unificada contenida en la sentencia de 1-12-03 (recurso 8/3569/02) que en la interpretación de otro precepto, ahora del artículo 131 de la LGSS fija como plazo de prórroga máxima no 30 meses sino nada menos que el tiempo que medie hasta la calificación de la incapacidad permanente aunque sea superior a los 30 meses previstos en el número 2 del artículo 131-bis, plazo que se indica no va dirigido al interesado sino a la entidad gestora para tramitar el procedimiento de calificación de Incapacidad Permanente. Se recrea la que dijimos quiso el legislador derogar, la Invalidez Provisional.

\section{Sobre la incompatibilidad de la prestación de Incapacidad Per- manente Total y la realización de trabajos, y la medida de suspen- sión que acuerda el INSS}

La cuestión que se analiza estriba en determinar si la entidad gestora responsable del abono de la prestación de incapacidad o invalidez permanente total, y que continúa desempeñando servicios puede decla- 
rar la incompatibilidad y, acordar la suspensión de la I.P. que tiene reconocida.

El artículo 141 de la LGSS establece que la pensión de Invalidez Permanente Total para la profesión habitual es compatible con el salario que el beneficiario pueda percibir en la misma empresa o en otra distinta con el alcance y en las condiciones que se determinen reglamentariamente. Por su parte, el desarrollo reglamentario del artículo 141-1 se contiene en el artículo 24 de la OM de 15 de abril de 1969 que admite la posibilidad de pacto de minoración del salario cuando la Incapacidad Permanente Total declarada «afecte a la capacidad exigida con carácter general, para desempeñar el nuevo puesto» en la misma o en otra empresa. Cuando se analiza qué pasa con quien es declarado en situación de Incapacidad Permanente Total pero pese a ello continúa desempeñando la misma profesión, los tribunales se encuentran con la inexistencia de previsión normativa. Pero no por ello se consiente esta situación en palabras del Tribunal Supremo «insólita». La solución se busca en la finalidad propia de la Incapacidad Permanente. La pensión de invalidez total tiene una función de sustitución de las rentas salariales que ya no se pueden obtener en el ejercicio de la profesión habitual. Ello comporta la compatibilidad con actividad distinta de la habitual, pero la incompatibilidad con la misma actividad respecto de la que se ha declarado la invalidez. Así viene a resolver la falta de previsión normativa, el Tribunal Supremo en su sentencia de fecha 18 de enero de 2002 (RJ 2002, 2681) (Ponente Sr. Martín Valverde).

Sin embargo, cuando analiza qué pasa con el inválido permanente total que viene desempeñando otro puesto (que incluso puede ser de la misma categoría o grupo profesional), el Tribunal Supremo llega a una solución radicalmente opuesta. Son exponente de esta doctrina las sentencias de fechas 28 de enero de 2002 (RJ 2002/3761) (ponente Sr. Fernández López), la de 30 de marzo de 2003 (EDJ 2003/11891), la de 28 de julio de 2003 (RJ 2003/7258) (Ponente, Sra. Calvo Ibarlucea) y la última de 27-1-05 (recurso 981/2004) (Ponente Sr. Fuentes López). En base a las mismas no existe norma que autorice la declaración de incompatibilidad y suspensión que, sólo está prevista en el artículo 18.4 de la OM de 18-1-96 que establece que: «si el procedimiento se hubiera iniciado en razón a que el perceptor de la pensión de invalidez estuviera ejerciendo trabajos por cuenta propia o ajena, y no se hubiera constatado error de diagnóstico o mejoría que justifique el reconocimiento del derecho de las prestaciones por invalidez permanente, en un grado de incapacidad inferior o la aptitud para trabajar, la Dirección provincial del INSS actuará de conformidad con la normativa en vigor $y$, en función de la incompatibilidad que pueda existir entre el percibo 
de la pensión y el trabajo desarrollado, dando lugar a la suspensión, cuando la actividad laboral exceda de los límites permitidos por el artículo 141.2 de la LGSS».

La remisión al artículo 141.2 que como es conocido se refiere a la posibilidad de compatibilizar el percibo de las prestaciones de Incapacidad Permanente Absoluta y Gran Invalidez», ha llevado, en palabras del Tribunal Supremo, a concluir que sólo existe previsión normativa para acordar la incompatibilidad y, correlativa suspensión, en los supuestos de IPA y GI. No existe previsión normativa de rango alguno que permita al INSS de oficio, acordar en otro caso la suspensión. Ante la laguna normativa y con una diferencia de 10 días el Tribunal Supremo forma una doctrina no sólo diferente sino incluso contradictoria; en la de 18-1-02 acude ante el vacío normativo a la finalidad de la Incapacidad, mientras que en la de 28-1-02 obvia la finalidad y, resuelve ateniéndose exclusivamente al texto de la norma. Esta solución ni siquiera se altera cuando se analiza el supuesto, pero referido a trabajadores autónomos para los que el artículo 82 de la Orden de 24 de septiembre de 1970 , por la que se dictan normas para la aplicación y desarrollo del régimen especial de la Seguridad Social de los trabajadores por cuenta propia o autónomos, al establecer el régimen de incompatibilidades de las pensiones vitalicias por I.P. - incluida por tanto la correspondiente al grado de total- establece el mismo tenor que el artículo 141-2 de la LGSS. No concurre pues la premisa, laguna legal, que justifica la doctrina unificada, en todo caso habrá que esperar a la respuesta del legislador.

Como ya anticipamos al inicio de estas notas, la regulación de esta prestación sí que se ve alterada por las reformas en las otras prestaciones. Como regla general se fija la prohibición de reconocimiento y revisión de la incapacidad cuando el beneficiario ha superado la edad de 65 años. Como excepción podrá reconocerse incapacidad a los trabajadores que retrasen la edad de jubilación más allá de los 65 años y, cuando la causa originaria de la I.P. derive de accidente de trabajo o enfermedad profesional (artículo 138 de la LGSS, tras el artículo 8 de la Ley 35/02 y 10 del RD 1132/02).

El reconocimiento de una pensión de I.P. será posible igualmente cuando se trate de jubilación flexible en las mismas condiciones que en el supuesto de retraso en la jubilación. Estas limitaciones, sin embargo, no juegan en el caso de la jubilación parcial con contrato de relevo (art. 14 del Decreto 1.131/02) en que podrá declararse una incapacidad permanente y revisarse, sea por causa común o por causa profesional, si bien será incompatible con la jubilación.

En los supuestos de reconocimiento de prestación de I.P. por con- 
tingencias comunes cuando el beneficiario no reúna los requisitos para acceder a la pensión de jubilación en el sistema de la Seguridad Social, la Ley 35/02 recorta la cobertura. El porcentaje, que fija la cuantía, no será el propio de la pensión de I.P. sino que se fija en el 50\%, coincidente con el mínimo de jubilación. Recorte que es asimismo significativo cuando de grandes inválidos se trata (50\% sobre el $50 \%$ y no sobre el $100 \%)$.

Por último unas breves notas sobre los cambios introducidos en la regulación de los regímenes especiales de autónomos y de los trabajadores por cuenta propia y que, como anticipamos, han afectado a esta prestación.

La reforma plasmada en la Ley 52/02, de 30 de diciembre, RD $1.273 / 03$, de 10 de octubre y, Ley 36/03, de 11 de noviembre extiende, en primer lugar, la prestación de Incapacidad Permanente Parcial al RETA cuando derive de enfermedad profesional. Pese a la expresión utilizada, no ha supuesto una homogeneización con el régimen general sino que se define esta IPP de forma distinta. Ya no es la que ocasiona a los trabajadores una disminución superior al 33\%, sino inferior al 50\% de su rendimiento normal para su profesión sin impedirle la realización de las tareas fundamentales de aquella. Sí que supone una equiparación con la cobertura que se presta en el Régimen general la posibilidad que establece el artículo 4 del RD 1.273/03 para los beneficiarios de IPT y menores de 60 años de optar por recibir una cantidad a tanto alzado equivalente a 40 mensualidades de la base reguladora, en los mismos términos que en el Régimen General.

Como última «mejora» se extiende la I.P. Total cualificada al RETA y a los Regímenes por cuenta propia de los regímenes especiales agrario y del mar. E1 RD 463/03, de 25 de abril, condiciona el reconocimiento de la Total «cualificada» a que se cumpla el requisito de la edad - igual o mayor a 55 años-, que el pensionista no ejerza una actividad retribuida por cuenta ajena o por cuenta propia y, se añade que el pensionista no ostente la titularidad de un establecimiento mercantil o industrial, ni de una explotación agraria o marítimo pesquera como propietario o arrendatario, usufructuario u otro concepto análogo. Requisito incongruente con la posibilidad de mantener la titularidad de un negocio o industria con la pensión de jubilación, lo que es aún más relevante si tenemos en cuenta cómo la pensión de incapacidad se convierte en jubilación después de los 65 años. 


\section{Bibliografía y artículos consultados}

Informes Estadísticos del Servicio Jurídico de la Administración de la Seguridad Social.

FERNÁNDEZ BERMÚdEZ, Juan (Letrado de la Seguridad Social). «Arbitrio judicial versus positivismo jurídico». Comunicación a las VII Jornadas de la Asociación Profesional del Cuerpo Superior de Letrados de la Administración de la Seguridad Social.

Luján, José. Profesión habitual a efectos de calificación de la Incapacidad Permanente Total.

Pérez Rodríguez, José M.a (Letrado de la Seguridad Social). «La Revisión Jurisdiccional de los Procedimientos en materia de Incapacidades Laborales». Comunicación a las VIII Jornadas de la Asociación Profesional de Letrados de la Administración de la Seguridad Social.

Comentario Sistemático a la Legislación reguladora de las pensiones. Ed. Lomazas Prenada 2004. 